\title{
Treatment at a high-volume academic research program mitigates racial disparities in pancreatic adenocarcinoma
}

\author{
Quyen D. Chu ${ }^{1}$, Mei-Chin Hsieh ${ }^{2}$, John F. Gibbs ${ }^{3} \wedge$, Xiao-Cheng Wu ${ }^{2}$ \\ ${ }^{1}$ Department of Surgery, LSU Health Sciences Center-Shreveport, Shreveport, LA, USA; ${ }^{2}$ Department of Louisiana Tumor Registry \& \\ Epidemiology and School of Public Health at LSU Health Sciences-New Orleans, New Orleans, LA, USA; ${ }^{3}$ Department of Surgery, Hackensack \\ Meridian School of Medicine, Nutley, NJ, USA \\ Contributions: (I) Conception and design: All authors; (II) Administrative support: None; (III) Provision of study materials or patients: None; (IV) \\ Collection and assembly of data: All authors; (V) Data analysis and interpretation: All authors; (VI) Manuscript writing: All authors; (VII) Final \\ approval of manuscript: All authors. \\ Correspondence to: John F. Gibbs, MD, MHCM. Hope Tower Oncology Administration, 19 Davis Ave., Neptune, NJ 07731, USA. \\ Email: John.Gibbs@hmhn.org.
}

Background: Racial disparities have long been a subject of concern between patients afflicted with pancreatic cancer in the United States. We believe that, in addition to a high-volume center, treatment at an academic research program (ARP) will mitigate racial outcome disparities.

Methods: A total of 12,950 patients diagnosed with stage I-III pancreatic adenocarcinoma from 2003-2011 and at ACS Commission on Cancer (COC) accredited facilities [e.g., high-volume ( $\geq 12$ cases/year) ARPs] were evaluated from the National Cancer Data Base (NCDB). Sociodemographic, clinicopathological, and treatment variables were compared between Black $(\mathrm{N}=1,127)$ and White $(\mathrm{N}=11,823)$ patients. The KaplanMeier Estimator and Cox Proportional Hazards Model were used for survival analysis. P value $\leq 0.05$ was considered significant.

Results: Black patients had a significantly higher overall survival (OS) than White patients, despite having a significantly lower household income, lower education level, more stage III disease, more Medicaid recipients, and higher comorbidity index $(\mathrm{P}<0.05)$. The 5 -year unadjusted OS $(28.6 \%$ versus $23.9 \%$, a median survival time (months) was (25.2 versus 23.7 months for Black and White patients, respectively $(\mathrm{P}<0.05)$. There was no significant difference in surgical margin status or receipt of chemoradiation between the two cohorts. After adjusting for covariates, race was no longer a significant predictor of OS $(\mathrm{P}=0.096)$.

Conclusions: Treatment at a high volume, ARP can mitigate racial disparities in pancreatic cancer.

Keywords: Pancreatic cancer; outcome; facility type; volume

Submitted Dec 10, 2020. Accepted for publication Sep 29, 2021.

doi: 10.21037/jgo-20-584

View this article at: https://dx.doi.org/10.21037/jgo-20-584

\section{Introduction}

Pancreatic ductal adenocarcinoma (PDAC) is a challenging disease with one of the poorest outcomes among all malignancies. Although it comprises only $3 \%$ of cancer diagnoses, it contributes to $7 \%$ of cancer deaths (1). PDAC is the fourth leading cause of cancer-related deaths in the U.S., and unfortunately, it is expected to become the second-leading cause of cancer death by 2030 (1-3). It is estimated that in 2020, 57,600 people will be diagnosed with the disease, of whom 47,050 will die of it (4).

Despite recent advances in prevention, diagnosis, and treatment for this disease, health care inequities remain,

^ORCID: 0000-0003-0037-8014. 
leading to disparities in outcomes between White and Black patients. It is well-established that Black patients with pancreatic cancer have worse survival rates than their White counterparts (5-9). The reasons behind such a disparity are multifactorial, including genetic, socioeconomic, and environmental factors.

A volume-outcome relationship has been demonstrated for many diseases and pancreatic cancer is no exception; high-volume centers tend to have better outcomes than low volume centers (10-12). Data suggest that Black patients are more likely to receive surgery at low-volume centers and/or by lower-volume surgeons (13). Disparities based on hospital characteristics might partly explain the poorer outcomes among Black patients.

We recently reported that OS from pancreatic adenocarcinoma was associated not only with volume but also with facility type (14). In other words, the outcome was dependent on where the patient received his/her treatment. Using the large National Cancer Data Base (NCDB) of over 33,000 patients, we found that patients who were treated at an academic research program (ARP) had the highest 5 -year OS (16.6\%) compared to comprehensive community cancer program $(13.2 \%$; CCCP) and community cancer program $(11.2 \%$; CCP; $\mathrm{P}<0.00001)$. Treatment at an ARP was an independent predictor of outcome after adjusting for gender, race/ethnicity, geography, hospital volume, primary payer status, income level, education level, comorbidity index, type of surgical resection, surgical margin status, histologic grade, lymph node status, number of lymph nodes retrieved, stage, and receipt of chemoradiation therapy. Compared to an ARP, those who received their treatment at a CCP had a $22 \%$ higher risk of mortality and those treated at a CCCP had a $5 \%$ increased risk of mortality (14).

Given the survival advantages of ARP, we sought to determine whether parity in outcomes between Black and White pancreatic cancer can be achieved, and if so, identified factors that may have contributed to such parity. This becomes an important endeavor since the overarching goal, from a public policy perspective, is not only to identify factors that lead to disparities in outcomes, but also those factors that achieve parity, irrespective of biological/ sociodemographic differences. Thus, the purpose of this study was to utilize a large national database to identify characteristics that lead to parity in outcomes between Black and White patients who underwent surgical resection for their pancreatic adenocarcinoma. To our knowledge, this is the first study to determine whether performance of high volume surgery at a specific facility type (i.e., ARP) will achieve racial parity for patients with pancreatic cancer. The significance of such a finding is to provide major stakeholders a better and deeper understanding of how to construct public policies to improve outcomes for the socioeconomically disadvantaged population with pancreatic adenocarcinoma.

We present the following article in accordance with the STROBE reporting checklist (available at https://dx.doi. org/10.21037/jgo-20-584).

\section{Methods}

\section{Data source}

The NCDB is a joint project of the Commission on Cancer (CoC) of the American College of Surgeons and the American Cancer Society with more than 1,500 CoCaccredited facilities contributing to the database. The data sets in the Participant Use Data File (PUF) were de-identified and were in compliance with the privacy requirements of the Health Insurance Portability and Accountability Act (HIPAA). The study was exempted from Institutional Review Board (IRB) approval by the Louisiana State University Health Sciences Center-Shreveport.

The study was conducted in accordance with the Declaration of Helsinki (as revised in 2013).

\section{Study population}

A cohort of 12,950 patients with stage I-III pancreatic adenocarcinoma (ICD-0-3; C25.0-C25.9) who were diagnosed from 2003 to 2011 and received pancreatic cancer surgery at COC-accredited program was analyzed to determine significant factors associated with OS. Annual hospital volume (AHV) were grouped as $<5$ patients, 5-9 patients, 10-19 patients, and greater than 20 patients (15). Patients were staged based on the 7th edition of the AJCC/ TNM staging system (16). Facility location was categorized into regions within the U.S.: (I) New England, (II) MidAtlantic, (III) South Atlantic, (IV) East North Central, (V) East South Central, (VI) West North Central, (VII) West South Central, (VIII) Mountain, and (IX) Pacific.

Race was limited to White and Black. Insurance status was classified as uninsured, private insurance/managed care, Medicaid, Medicare, and other governmental insurance. Patients with both private insurance and Medicare were grouped in the private insurance category. Median household income level was classified as (I) $<\$ 38,000$, (II) 
$\$ 38,000-\$ 47,999$, (III) $\$ 48,000-\$ 62,999$, and (IV) $\geq \$ 63,000$. Education level was classified into percentage of adults (age $\geq 25$ years) who did not graduate from high school in the area based on the 2012 American Community Survey data: (I) $\geq 21 \%$, (II) $13-20.9 \%$, (III) $7-12.9 \%$, and (IV) $<7 \%$. Charlson/Deyo comorbidity score was reported as 0,1 or 2 $(17,18)$.

Population density was classified as (I) metropolitan with $\geq 1$ million, (II) metropolitan with 250,000 to 1 million, (III) metropolitan with $<250,000$, (IV) urban and (V) rural with $<2,500$. Great circle distance (miles is defined as the distance between the patient's residence at diagnosis and the hospital that reported the case.

\section{Statistical analysis}

The nonparsimonious approach using variables such as age, race, etc. was used to construct models. Descriptive statistics for the predictor variables by race, and interquartile range (IQR), where appropriate, were reported and Chi-square test was used to assess the unadjusted association. The Kaplan-Meier method was used to estimate survival time by race and its statistically significant difference was assessed using the log-rank test. Univariate Cox proportional hazards regression model was used to identify factors associated with the risk of deaths for all causes. The multivariable Cox proportional hazards regression analysis was used to determine independent significant factors associated with the risk of death for all causes, and the hazard ratios (HR) and $95 \%$ confidence intervals (CI) were calculated. A P value $\leq 0.05$ was considered statistically significant. All statistical analyses were performed using SAS Version 9.4 statistical software, (SAS Institute Inc., Cary, NC, USA).

\section{Results}

The median follow-up duration was 20 months (IQR, 24 months). Summary statistics on patient characteristics and treatment outcomes are shown in Table 1. The median age of the entire cohort was 66 years (IQR, 16 years).

There were significantly more White males $(52 \%$ vs. $43 \%)$ and Black females $(57 \%$ vs. $48 \%)(\mathrm{P}<0.0001)$. Note that most patients were White (91\%). Compared to White patients, Black patients present at a significantly younger age $(\mathrm{P}<0.0001)$, have lower level of income $(\mathrm{P}<0.0001)$ and education $(\mathrm{P}<0.0001)$, more likely to be uninsured and/or recipient of Medicaid $(\mathrm{P}<0.0001)$. Additionally, Black patients were found to reside in metropolitan area $\geq 1$ million $(\mathrm{P}<0.0001)$, reside at great circle distance $<$ 50 miles $(\mathrm{P}<0.0001)$, diagnose at early stage $(\mathrm{P}<0.0001)$, have well differentiated grade $(\mathrm{P}<0.0001)$, and have higher comorbidity index $(\mathrm{P}=0.001)$. There is a significant association between a predictor (such as stage) and outcome variable (race), but we do not know which group. There was no difference in the rate of achieving negative surgical margins $(\mathrm{P}=0.199)$ and receipt of chemotherapy $(\mathrm{P}=0.27)$ and radiation therapy $(\mathrm{P}=0.88)$.

Figure 1 shows the Kaplan-Meier OS curve by race. The 5 -year OS rate was $23.9 \%$ (95\% CI: $22.9-24.8 \%$ ) for White patients and 28.6\% (95\% CI: 25.3-31.9\%) for Black patients $(\mathrm{P}=0.0149)$. The median survival was 23.7 months (95\% CI: 23.1-24.3 months) for White and 25.2 months (95\% CI: 22.8-28.1 months) for Black patients.

Table 2 presents the unadjusted and adjusted HRs and corresponding $95 \%$ CIs to identify factors associated with overall survival. In the unadjusted model, race, gender, age, income level, education level, insurance status, stage of disease, grade, great circle distance, type of surgical resection, margin status, and comorbidities were factors associated with outcomes. We have performed analyses of low-volume centers and at facility type level. We found there was no significance difference in survival between racial groups treated at low volume $(\leq 10)$ in CCP or CCCP and at each facility type (Tables S1,S2).

\section{Unadjusted factors associated with overall survival}

Interestingly enough, White patients have a $10 \%$ increased risk of dying compared to Black patients; males have a $7 \%$ increased risk of dying compared to females; patient's ages $\geq 55$ years have a $32 \%$ to $86 \%$ increased risk of dying compared to those ages $<55$ years (Table 2). Those with an annual income $<\$ 63,000$ had a $12 \%$ to $21 \%$ risk of dying compared to those with an annual income $\geq \$ 63,000$. Patients with limited education level had a $7 \%$ to $19 \%$ risk of dying compared to those with the highest level of education. Medicaid recipients are at a $27 \%$ risk of dying compared to privately insured patients and Medicare recipients are at a $39 \%$ risk of dying compared to privately insured patients. Pathologic stage 2 disease have 2.54 times risk of dying and patients with stage 3 disease have 3.45 times risk of dying compared to those with stage I disease, respectively. Compared to well-differentiated tumors, those with moderately differentiated tumors are 2.64 times more likely to die, while those with poorly/ undifferentiated tumors are 3.58 times more likely to die. 
Table 1 Demographic and clinical characteristics of stage I-III pancreatic cancer patients who received a pancreatectomy at high-volume academic research program

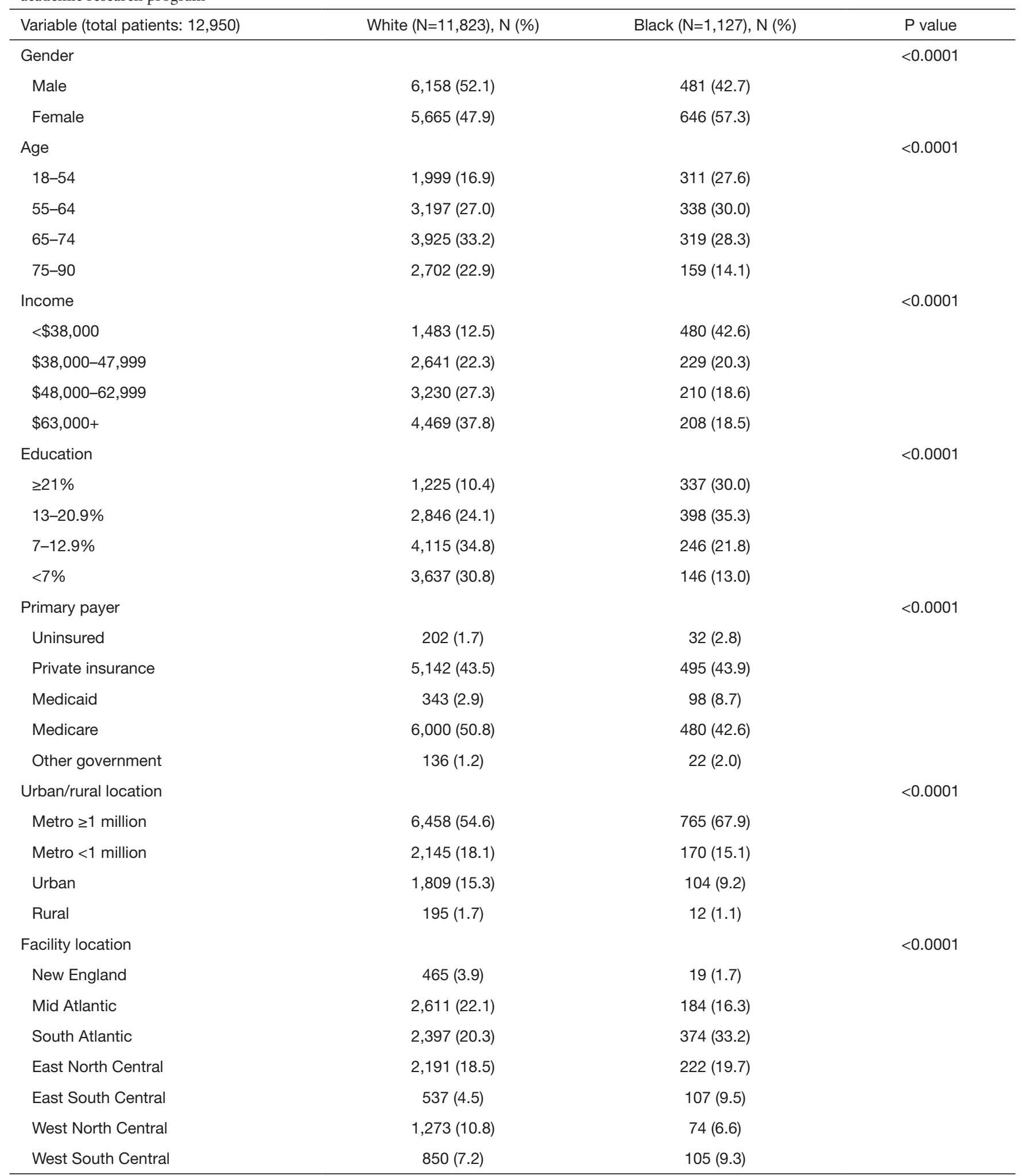

Table 1 (continued) 
Table 1 (continued)

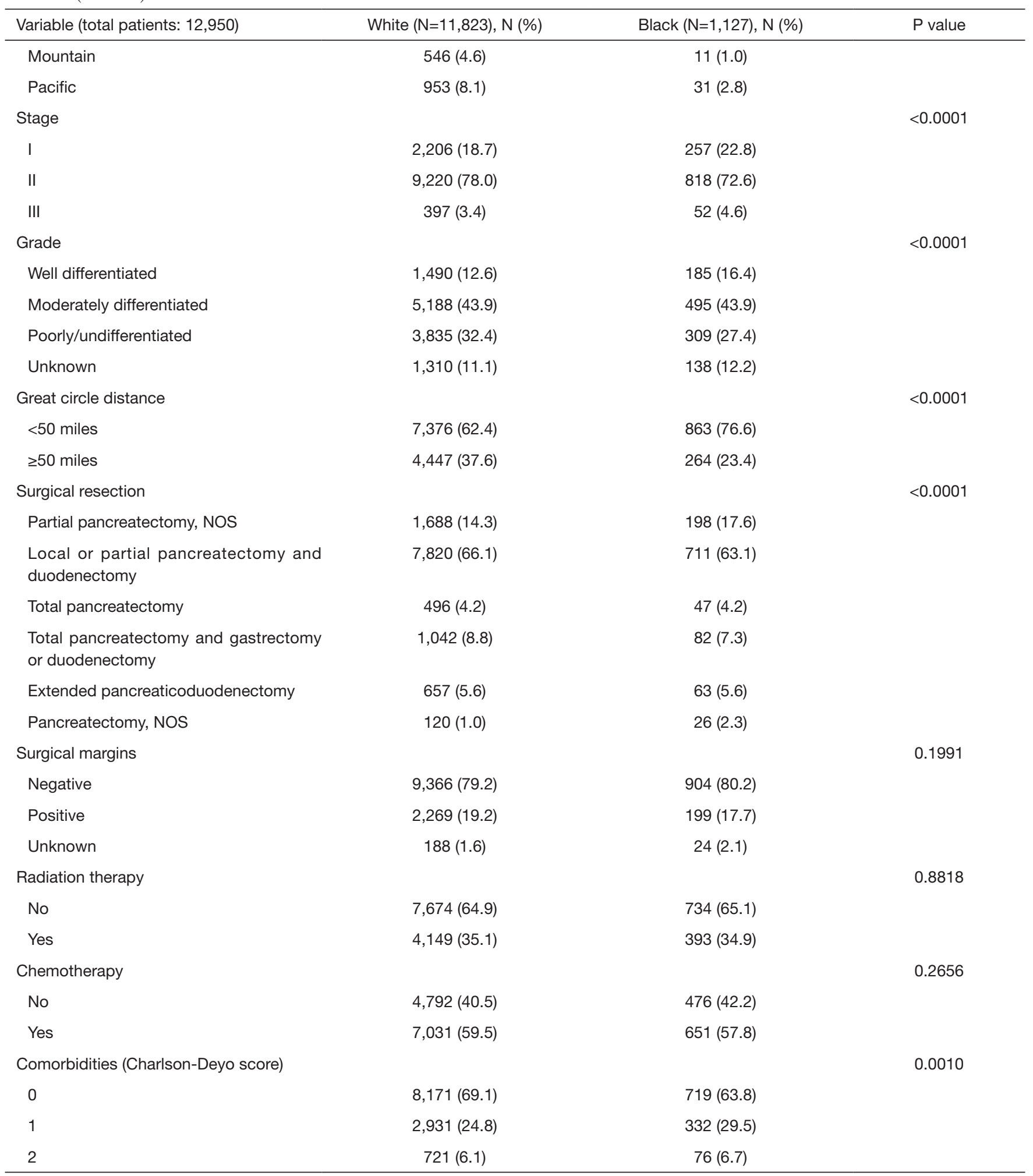

Education is classified as the percentage of adults who did not graduate from high school. 


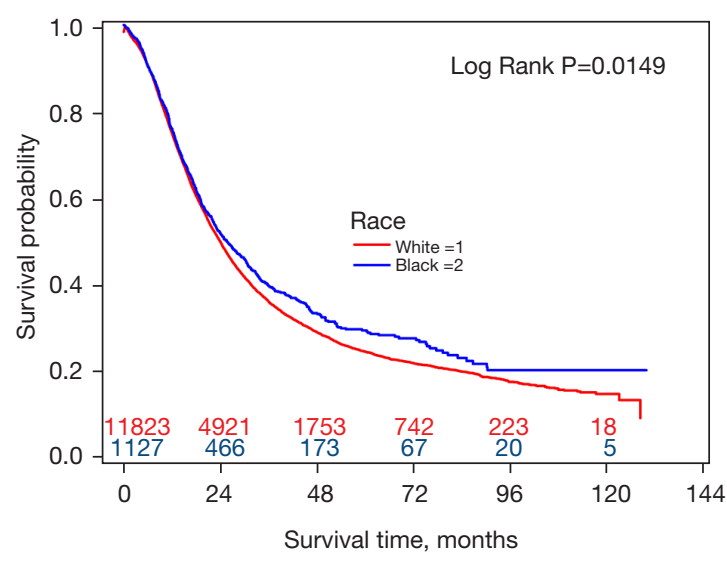

Figure 1 Kaplan-Meier overall survival curve for stage IIII pancreatic adenocarcinoma based on race. The 5 -year OS rate was $23.9 \%$ (95\% CI: $22.9-24.8 \%$ ) for White and $28.6 \%$ (95\% CI: $25.3-31.9 \%$ ) for Black Stage I-III pancreatic cancer patients $(\mathrm{P}=0.0149)$. The median survival was 23.7 months $(95 \%$ CI: 23.1-24.3 months) for White and 25.2 months (95\% CI: 22.8-28.1 months) for Black patients. CI, confidence interval.

The presence of positive surgical margins has an $83 \%$ risk of dying compared to those with negative surgical margins. Finally, those with a Charles-Deyo comorbidity index of 1 are at a $10 \%$ increased risk of dying compared to those with a 0 index and those with an index of 2 are at a 36\% risk of dying compared to those with an index of zero.

\section{Adjusted factors associated with overall survival}

After adjusting for patient socioeconomic status, tumor characteristics, and treatment, race no longer remains a significant predictor of outcome $(\mathrm{P}=0.0963)$. Independent predictors of OS were gender, age, income level, primary payer status, Stage of disease, histology, comorbidity index, type of surgical resection, surgical margin status, and receipt of chemotherapy. Males have a $5 \%$ increased risk of mortality compared to females. Patient's ages $\geq 55$ years have a $22 \%$ to $48 \%$ increased risk of dying compared to those ages $<55$ years. An annual household income $<\$ 63,000$ had an $11 \%$ to $19 \%$ risk of dying compared to those with an annual income $\geq \$ 63,000$. From insurance status, Medicaid recipients are at a $24 \%$ risk of dying compared to privately insured patients and Medicare recipients are at a $14 \%$ risk of dying compared to privately insured patients. Pathologic stage 2 disease have a 2.05 -fold risk of dying and patients with stage 3 disease have a 2.86-fold risk of dying compared to those with stage I disease, respectively. Compared to well-differentiated tumors, those with moderately differentiated tumors are 2.27 times more likely to die, while those with poorly/undifferentiated tumors are 3.08 times more likely to die. Positive surgical margins were associated with a $61 \%$ risk of dying compared to those with negative surgical margins. Patients who did not receive chemotherapy are at a $36 \%$ risk of dying compared to those who received chemotherapy. Finally, those with a CharlesDeyo comorbidity index of 1 are at a $6.5 \%$ increased risk of dying compared to those with a 0 index and those with an index of 2 are at a $28 \%$ risk of dying compared to those with a 0 index.

\section{Discussion}

Significant progress in the medical field has been made over the past decades, but not everyone has benefitted equally from it. It is well-established that Black patients with pancreatic cancer have lower overall survival than their White counterparts $(5,7-9,19-21)$. It is estimated that survival among Black patients with pancreatic adenocarcinoma is $10 \%$ to $20 \%$ worse than their White counterparts $(5,7,8)$. Survival disparities may be due to differences in biology and socioeconomic status. The incidence of pancreatic cancer is $40 \%$ to $90 \%$ higher in the Black population, and the disease tends to present at a younger age, have more advanced disease at presentation, and more likely to belong to socioeconomically disadvantaged population (8,9,22-24).

A volume-outcome relationship for PDAC is wellestablished (10-12). Patients treated at high-volume centers tend to have better OS compared to those treated at lowvolume centers. Previous reports found that Black patients were more likely to be treated at low-volume centers compared to White patients, which might partly explain the outcome disparities between the two populations $(10,13)$. Epstein et al. found that among the 570 patients who had pancreatectomy, 36\% of White patients received their surgery at high volume centers with a high-volume surgeon, compared to only $10 \%$ of Black patients. Such differences in the rates of surgery at high volume centers with high volume surgeons were statistically significant, even after adjusting for other variables (13). Similarly, Eppsteiner et al. found that compared to Black patients, White patients were more likely to have their resection performed by high volume surgeons and at a teaching hospital; high volume surgeons had a significantly lower mortality compared to 
Table 2 Factors associated with overall survival in stage I-III pancreatic cancer patients who received a pancreatectomy at high-volume academic research program

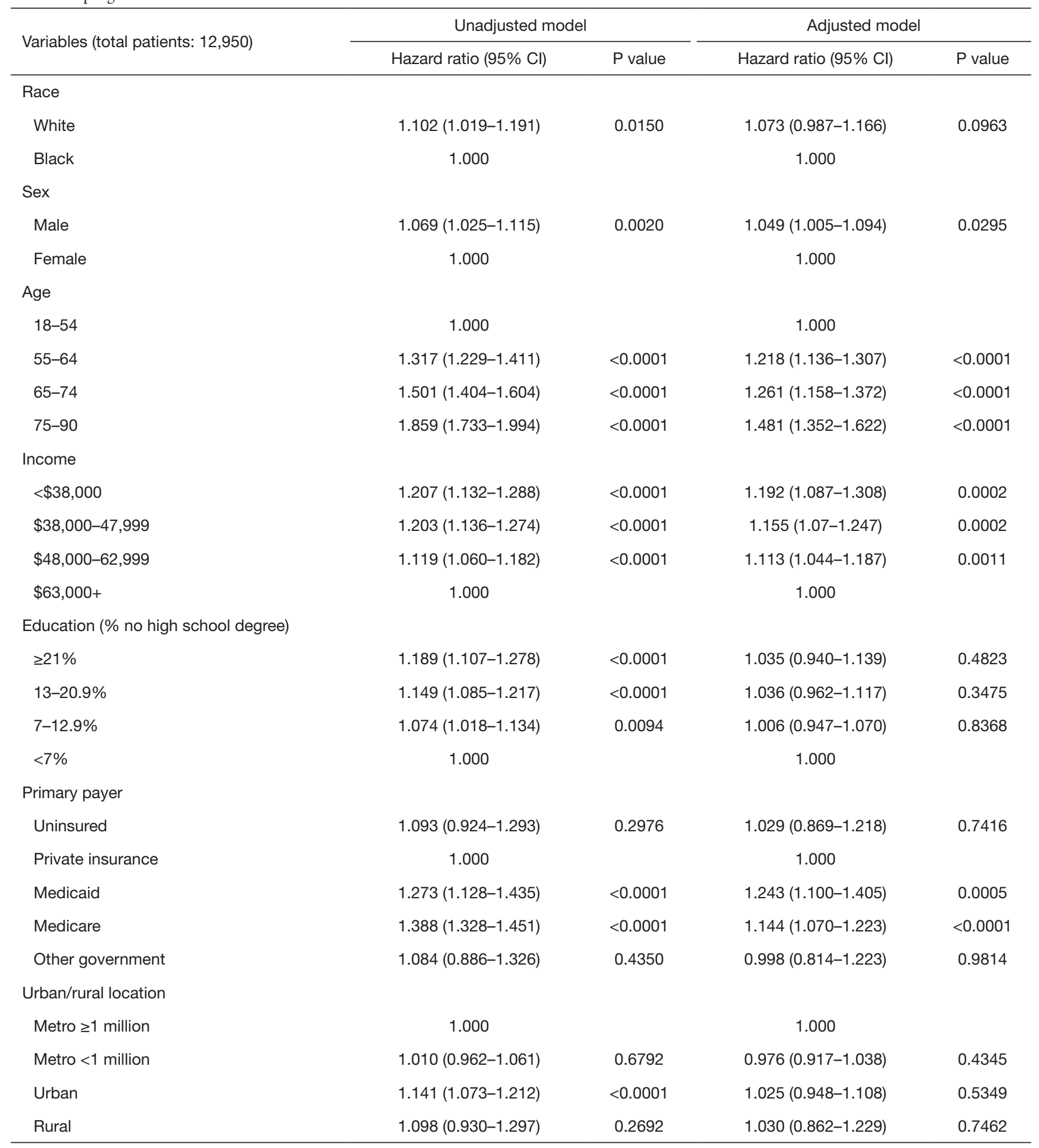

Table 2 (continued) 
Table 2 (continued)

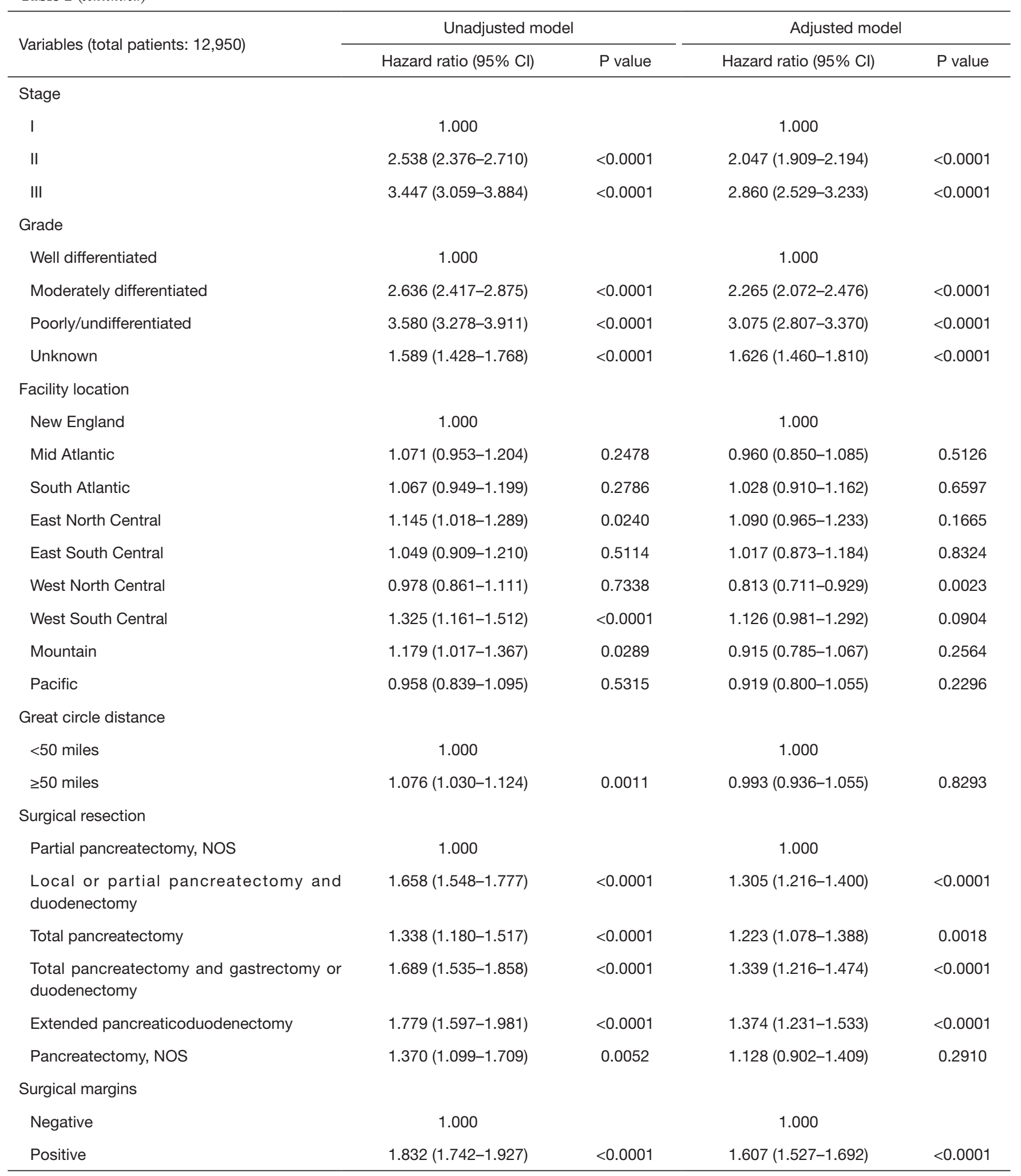

Table 2 (continued) 
Table 2 (continued)

\begin{tabular}{|c|c|c|c|c|}
\hline Variables (total patients: 12,950$)$ & \multicolumn{2}{|c|}{ Unadjusted model } & \multicolumn{2}{|c|}{ Adjusted model } \\
\hline Unknown & $1.373(1.168-1.613)$ & 0.0001 & $1.386(1.177-1.631)$ & $<0.0001$ \\
\hline \multicolumn{5}{|l|}{ Radiation therapy } \\
\hline No & $1.029(0.985-1.075)$ & 0.2016 & $1.023(0.969-1.081)$ & 0.4043 \\
\hline \multicolumn{5}{|l|}{ Chemotherapy } \\
\hline No & $1.021(0.977-1.066)$ & 0.3519 & $1.356(1.282-1.433)$ & $<0.0001$ \\
\hline Yes & 1.000 & & 1.000 & \\
\hline \multicolumn{5}{|c|}{ Comorbidities (Charlson-Deyo score) } \\
\hline 2 & $1.360(1.250-1.481)$ & $<0.0001$ & $1.284(1.178-1.399)$ & $<0.0001$ \\
\hline
\end{tabular}

low volume surgeons (10).

Besides volume, we also found a facility-outcome relationship for PDAC (14). In our previous study, we found that OS for resected pancreatic adenocarcinoma was dependent on facility type. In that study, ARP had the highest OS compared to comprehensive community cancer program (CCCP) and community cancer program (CCP), even after adjusting for volume as well as other socioeconomic variables. Compared to ARP, the likelihood of death was $5 \%$ higher at CCCP and $22 \%$ higher at CCP $(\mathrm{P}<0.01)(14)$.

Given these facts, we sought to determine whether an optimal set of conditions exists, that could virtually eliminate disparate outcomes between the two racial/ ethnic groups, regardless of other socio demographic/ economics challenges. Thus, we restricted our study only to high volume centers and ARP. To our knowledge, this is the first study to combine volume and facility to determine the volume/facility-relationship in patients who underwent pancreatic surgery for PDAC.

Receipt of appropriate treatments have been demonstrated to contribute to the racial disparities in pancreatic cancer (7). Surgical resection is the only modality that offers potential cure, yet Black patients are less likely to receive such a treatment compared to other racial populations $(5,6,25-28)$. Such disparities may be related to lack of surgical evaluation at the point of the initial evaluation or afterward (5). Analyzing the Surveillance, Epidemiology, and End Results (SEER)-Medicare linked data, Riall and colleagues reported that $29 \%$ of Black patients with potentially resectable pancreatic cancers never received surgical evaluation (5). Furthermore, even after surgical evaluation has taken place, Black patients were still $32 \%$ less likely to undergo surgical resection. It is unknown whether this was due to the lack of surgical expertise on the surgeon's part and/or the lack of having appropriate infrastructure to successfully care for these patients from the hospital's perspective, or patient's refusal of offered surgical treatment $(5,28)$. Essentially, it is a question of a volume-outcome relationship at both the hospital level and individual surgeon level, and to address it, we therefore limited our study to only high-volume centers.

In this study, we found that race was no longer an independent predictor of outcome, an observation reported by others $(5,28)$. However, what is unique about our study is that Black patients had a better OS than their White counterparts. To our knowledge, this is the first study to demonstrate a "reverse" disparity along racial lines, favoring the Black pancreatic cancer population. Such a unique finding was observed despite Black patients being significantly more likely to belong to the lower income stratum, had lower education level, higher comorbidity index, more stage III disease, and more likely to receive Medicaid. This suggests that irrespective of 
socioeconomic challenges and biologic differences between the racial groups, outcome disparities can be mitigated if patients were treated at high volume ARP. Perhaps high-volume ARP are high-quality centers that provide appropriate coordination of care that is comprehensive, multidisciplinary, and evidence-based care, irrespective of a patient's socioeconomic background. Whether this was the case is unknown since such data were not collected in the database.

The socioeconomic demographics of our population are similar to those from other studies (8), mainly that Black patients with pancreatic cancer presented at a younger age, come mainly from socioeconomically challenged background, and have more aggressive disease.

The reported racial disparities might partly be explained by the disparities in receipt of chemotherapy and radiotherapy. In a study by Abraham et al., Black patients with resected cancers were $25 \%$ less likely to receive adjuvant chemotherapy and $30 \%$ less likely to receive adjuvant chemoradiation therapy than White patients (29). Similarly, Wray et al., found a $10 \%$ reduction in the rates of receipt of chemotherapy among Black compared to White patients, although the rates of receipt of radiotherapy was similar among the two racial groups (19). However, in our study, we found no differences in the receipt of chemotherapy and/or radiotherapy between Black and White pancreatic cancer patients. This suggests that high volume ARP centers have the ability not only to perform the complex surgery, but also possess the infrastructure to ensure that patients receive appropriate chemoradiation therapy.

Margin status plays a critical role in predicting outcomes $(14,28,30,31)$. In our study, margin status was an independent predictor of outcome. A positive margin status following a resection portends a $61 \%$ increase in mortality compared to a negative margin status. Thus, the poor outcomes observed in Black pancreatic cancer cohort may be related to a higher rate of positive margin. In our study, we found no significant difference in margin status between Black and White pancreatic cancer patients, and this may contribute to the parity in outcome between the two groups. Perhaps, a high-volume ARP centers have the expertise to achieve a higher rate of negative margins. In our previous study, we found that ARP had a significantly higher rate of negative margin status compared to CCCP and CCP (14).

Our study does have several limitations. The limitation of race to black or white due to the small sample size for other races in the rural area may influence the generality of the findings to the black $(\mathrm{N}=1,127)$ and white $(\mathrm{N}=11,823)$ population. A question will arise whether the number variation between white and black has any effect. We included all eligible Blacks and White patients in this study. Because we did not include other races and Hispanics due to small numbers of patients, the findings only apply to Black and White patients. We did not do any sampling. All eligible patients in the NCDB database were included. Because most high-volume ARPs are included in the NCDB, the findings are generalizable. The HRs of black $v s$. white are close to 1.00 and $95 \%$ CIs are not very wide. Patients may have visited more than one facility making it difficult to delineate the true impact of facility type is a potential limitation. Although the data can be interpreted as "reversed disparity "it is possible that a sex by treatment bias is present. Referral patterns and patient's treatment center selection preference is not captured in the database. Additionally, the study does not account for possible migration of surgeons moving from one facility type to another, as well as the exact adjuvant chemotherapy protocol or adjuvant chemo-radiotherapy protocol following surgery. An interesting and unresolved question in this study is whether a racial difference exists between patients receiving neoadjuvant versus adjuvant therapy. We could not address this question as the database utilized for this study does not distinguish between neoadjuvant nor adjuvant chemotherapy. The study also only looks at those who had surgery. Therefore, information on other aspects of racial/ethnic disparities (i.e., access to surgery) cannot be extrapolated based on these data. Our analysis lacks causespecific survival information since the cause of death was not captured in NCDB. Finally, testing many predictors may result in a multiple testing problem introducing in type I errors.

\section{Conclusions}

We report a volume/facility-outcome relationship for patients who underwent pancreatectomy for PDAC. Our study demonstrates that poor outcomes are not an inevitability for Black patients with stage I-III PDAC. Unlike previous investigators, our study demonstrated that Black patients actually had better overall survival than their White counterpart when treatment was done at a highvolume ARP center. To our knowledge, this is the first study to report this observation. Such survival advantage was achieved despite many Black patients belonging to a 
socioeconomically disadvantaged population. Although OS was worse for White pancreatic cancer patients, race was not an independent predictor of outcome after adjusting for other variables. This suggests that parity in outcome between the two racial populations can be achieved and that efforts should go towards better understanding, at a granular level, the factors that allowed high volume ARPs to mitigate racial disparities in pancreatic cancer outcome. We plan to explore whether the failure to rescue at low volume centers is associated with the improved outcomes from all ARPs with high volumes.

\section{Acknowledgments}

This paper was presented at the Annual Meeting of the 2020 Americas-Hepato-Pancreato-Biliary Association, March 5-8, 2020, Loews Miami Beach Hotel, Miami Beach, FL, USA. The data used in the study are derived from a deidentified NCDB file. The American College of Surgeons and the Commission on Cancer have not verified and are not responsible for the analytic or statistical methodology employed, or the conclusions drawn from these data by the investigator. The authors are indebted to the faculty and learners from the Jefferson College of Population Health for their valuable input during discussions.

Funding: This work was supported by the Edward \& Freda Green Professorship in Surgical Oncology.

\section{Footnote}

Reporting Checklist: The authors have completed the STROBE reporting checklist. Available at https://dx.doi. org/10.21037/jgo-20-584

Peer Review File: Available at https://dx.doi.org/10.21037/ jgo-20-584

Conflicts of Interest: All authors have completed the ICMJE uniform disclosure form (available at https://dx.doi. org/10.21037/jgo-20-584). JFG reports honoraria received for NJHA Webinar: EHR in the Management of Chronic Disease on 06-28-2021. JFG serves as an unpaid editorial board member of Journal of Gastrointestinal Oncology from fanuary 2021 to December 2022. The other authors have no conflicts of interest to declare.

Ethical Statement: The authors are accountable for all aspects of the work in ensuring that questions related to the accuracy or integrity of any part of the work are appropriately investigated and resolved. The study was exempted from Institutional Review Board (IRB) approval by the Louisiana State University Health Sciences CenterShreveport, and was conducted in accordance with the Declaration of Helsinki (as revised in 2013).

Open Access Statement: This is an Open Access article distributed in accordance with the Creative Commons Attribution-NonCommercial-NoDerivs 4.0 International License (CC BY-NC-ND 4.0), which permits the noncommercial replication and distribution of the article with the strict proviso that no changes or edits are made and the original work is properly cited (including links to both the formal publication through the relevant DOI and the license). See: https://creativecommons.org/licenses/by-nc-nd/4.0/.

\section{References}

1. Siegel RL, Miller KD, Jemal A. Cancer statistics, 2016. CA Cancer J Clin 2016;66:7-30.

2. Rahib L, Smith BD, Aizenberg R, et al. Projecting cancer incidence and deaths to 2030: the unexpected burden of thyroid, liver, and pancreas cancers in the United States. Cancer Res 2014;74:2913-21.

3. Siegel RL, Miller KD, Jemal A. Cancer statistics, 2019. CA Cancer J Clin 2019;69:7-34.

4. Siegel RL, Miller KD, Jemal A. Cancer statistics, 2020. CA Cancer J Clin 2020;70:7-30.

5. Riall TS, Townsend CM Jr, Kuo YF, et al. Dissecting racial disparities in the treatment of patients with locoregional pancreatic cancer: a 2-step process. Cancer 2010;116:930-9.

6. Bilimoria KY, Bentrem DJ, Ko CY, et al. National failure to operate on early stage pancreatic cancer. Ann Surg 2007;246:173-80.

7. Singal V, Singal AK, Kuo YF. Racial disparities in treatment for pancreatic cancer and impact on survival: a population-based analysis. J Cancer Res Clin Oncol 2012;138:715-22.

8. Khawja SN, Mohammed S, Silberfein EJ, et al. Pancreatic cancer disparities in African Americans. Pancreas 2015;44:522-7.

9. Heller DR, Nicolson NG, Ahuja N, et al. Association of Treatment Inequity and Ancestry With Pancreatic Ductal Adenocarcinoma Survival. JAMA Surg 2020;155:e195047.

10. Eppsteiner RW, Csikesz NG, McPhee JT, et al. Surgeon volume impacts hospital mortality for pancreatic resection. Ann Surg 2009;249:635-40. 
11. Al-Refaie WB, Muluneh B, Zhong W, et al. Who receives their complex cancer surgery at low-volume hospitals? J Am Coll Surg 2012;214:81-7.

12. McPhee JT, Hill JS, Whalen GF, et al. Perioperative mortality for pancreatectomy: a national perspective. Ann Surg 2007;246:246-53.

13. Epstein AJ, Gray BH, Schlesinger M. Racial and ethnic differences in the use of high-volume hospitals and surgeons. Arch Surg 2010;145:179-86.

14. Chu QD, Zhou M, Peddi P, et al. Influence of facility type on survival outcomes after pancreatectomy for pancreatic adenocarcinoma. HPB (Oxford) 2017;19:1046-57.

15. Categories of Accreditation. Available online: http://www. facs.org/cancer/coc/categories.html (Accessed November 9, 2014).

16. Edge SB, Compton CC. The American Joint Committee on Cancer: the 7 th edition of the AJCC cancer staging manual and the future of TNM. Ann Surg Oncol 2010;17:1471-4.

17. Charlson ME, Pompei P, Ales KL, et al. A new method of classifying prognostic comorbidity in longitudinal studies: development and validation. J Chronic Dis 1987;40:373-83.

18. Deyo RA, Cherkin DC, Ciol MA. Adapting a clinical comorbidity index for use with ICD-9-CM administrative databases. J Clin Epidemiol 1992;45:613-9.

19. Wray CJ, Castro-Echeverry E, Silberfein EJ, et al. A multi-institutional study of pancreatic cancer in Harris County, Texas: race predicts treatment and survival. Ann Surg Oncol 2012;19:2776-81.

20. Chang KJ, Parasher G, Christie C, et al. Risk of pancreatic adenocarcinoma: disparity between African Americans and other race/ethnic groups. Cancer 2005;103:349-57.

21. Silverman DT, Hoover RN, Brown LM, et al. Why do Black Americans have a higher risk of pancreatic cancer than White Americans? Epidemiology 2003;14:45-54.

22. Ward E, Jemal A, Cokkinides V, et al. Cancer disparities by race/ethnicity and socioeconomic status. CA Cancer J Clin 2004;54:78-93.

23. Eloubeidi MA, Desmond RA, Wilcox CM, et al. Prognostic factors for survival in pancreatic cancer: a population-based study. Am J Surg 2006;192:322-9.

24. Steyerberg EW, Earle CC, Neville BA, et al. Racial differences in surgical evaluation, treatment, and outcome of locoregional esophageal cancer: a population-based analysis of elderly patients. J Clin Oncol 2005;23:510-7.

25. Murphy MM, Simons JP, Hill JS, et al. Pancreatic resection: a key component to reducing racial disparities in pancreatic adenocarcinoma. Cancer 2009;115:3979-90.

26. Riall TS, Nealon WH, Goodwin JS, et al. Pancreatic cancer in the general population: Improvements in survival over the last decade. J Gastrointest Surg 2006;10:1212-23; discussion 1223-4.

27. Cress RD, Yin D, Clarke L, et al. Survival among patients with adenocarcinoma of the pancreas: a populationbased study (United States). Cancer Causes Control 2006;17:403-9.

28. Moaven O, Richman JS, Reddy S, et al. Healthcare disparities in outcomes of patients with resectable pancreatic cancer. Am J Surg 2019;217:725-31.

29. Abraham A, Al-Refaie WB, Parsons HM, et al. Disparities in pancreas cancer care. Ann Surg Oncol 2013;20:2078-87.

30. Strobel O, Hank T, Hinz U, et al. Pancreatic Cancer Surgery: The New R-status Counts. Ann Surg 2017;265:565-73.

31. Tummers WS, Groen JV, Sibinga Mulder BG, et al. Impact of resection margin status on recurrence and survival in pancreatic cancer surgery. Br J Surg 2019;106:1055-65.
Cite this article as: Chu QD, Hsieh MC, Gibbs JF, Wu $\mathrm{XC}$. Treatment at a high-volume academic research program mitigates racial disparities in pancreatic adenocarcinoma. J Gastrointest Oncol 2021;12(6):2579-2590. doi: 10.21037/jgo-20584 


\section{Supplementary}

Table S1 Hazard ratios and 95\% CIs for race at low volume non-academic research program facilities (volume $\leq 10$ and facility type in community cancer program or comprehensive community cancer program)

\begin{tabular}{lccccc}
\hline \multirow{2}{*}{ Race } & \multicolumn{2}{c}{ Unadjusted model } & & \multicolumn{2}{c}{ Adjusted model } \\
\cline { 2 - 3 } \cline { 5 - 6 } White & Hazard ratio $(95 \% \mathrm{Cl})$ & $\mathrm{P}$ value & Hazard ratio $(95 \% \mathrm{Cl})$ & $\mathrm{P}$ value \\
Black & $0.992(0.921-1.068)$ & 0.8244 & $0.977(0.903-1.057)$ & 1.000 \\
\hline
\end{tabular}

$\mathrm{Cl}$, confidence interval.

Table S2 Hazard ratios and 95\% CI for race stratified by facility type

\begin{tabular}{|c|c|c|c|c|}
\hline Race & \multicolumn{2}{|c|}{ Unadjusted model } & \multicolumn{2}{|c|}{ Adjusted model } \\
\hline \multicolumn{5}{|c|}{ All facilities } \\
\hline White & $1.040(0.9979-1.084)$ & 0.0676 & $1.005(0.960-1.052)$ & 0.8328 \\
\hline Black & 1.000 & & 1.000 & \\
\hline White & $0.889(0.738-1.070)$ & 0.2137 & $0.900(0.728-1.113)$ & 0.3292 \\
\hline Black & 1.000 & & 1.000 & \\
\hline \multicolumn{5}{|c|}{ Comprehensive community cancer program } \\
\hline White & $1.020(0.952-1.093)$ & 0.5708 & $0.980(0.910-1.056)$ & 0.5924 \\
\hline \multicolumn{5}{|c|}{ Academic/research program } \\
\hline White & $1.057(1.001-1.117)$ & 0.0470 & $1.024(0.964-1.088)$ & 0.4382 \\
\hline Black & 1.000 & & 1.000 & \\
\hline
\end{tabular}

$\mathrm{Cl}$, confidence interval. 